\title{
INNER THERMODYNAMIC EQUILIBRIA
}

\author{
BY J. E. TREVOR
}

\section{Introduction}

The differential of the energy of any material system of given masses subject only to reversible actions of heating and compression is represented as

$$
d \varepsilon=t d \eta-p d v
$$

where $p$ and $t$ denote the pressure and the absolute' or thermodynamic temperature, $\eta$ the entropy and $v$ the volume of the total mass. For, the two terms of the second member of this equation represent the heat and work added to the system, and by hypothesis these are the work equivalents of the only actions exerted upon it from without. The energy of such a system is accordingly a function of its entropy and volume and is determined by these. This however is not in general true of a physically and chemically homogeneons phase of a material system, for the energy of a phase changes with the variation of the masses of its constituents. The state of a phase, and consequently the value of its energy, is wholly determinate when for given entropy and volume the masses of its constituents are given, so, as has been remarked by $\mathrm{Gibbs}^{1}$, the energy of such a phase is a function of its entropy, its volume and the masses $m_{l}, m_{z}$. . . . $m_{\nu}$ of its several constituents, $\nu$ in number, and we can write

$$
d \varepsilon^{\prime}=t d \eta^{\prime}-p d v^{\prime}+\sum \mu d m^{\prime}
$$

where for any constituent $(i)$ the quantity $\mu_{i}$ is the mass derivative

$$
\mu_{i}=\frac{\partial \varepsilon}{\partial m_{i}}
$$

of the energy for this constituent (it is termed by Gibbs the potential of the latter) and the summation is extended over all the $v$ constituents. The constituents must be so chosen that the differen-

'J. W. Gibbs. Trans. Conn. Acad. 3, II6 (I875). 
tials of their masses are independent and can express every possible variation in the composition of the phase. The quantities $\varepsilon, \eta, v, m_{1}$, $m_{2}, \ldots m_{\nu}$ in (2) have accents to indicate that they relate to a single phase and not to a system of phases, the accents are omitted from $t, p, \mu_{r}, \mu_{2}, \ldots \mu_{v}$ for the reason that these quantities have uniform values throughout all the coexistent phases of a system in equilibrium. ${ }^{1}$

In Gibbs's great paper on the equilibrium of heterogeneous substances it is shown that, although the form of the relation holding among the energy, entropy, volume and masses of a phase is not known, yet an integral expression involving these independent variables together with the energy and its partial derivatives can be obtained by integrating (2) for constant state-i.e., for constant temperature, pressure and relative masses of the several constituents-from zero to a finite amount of phase. The operation is possible, because each potential, $\mu$, is a function of the temperature, pressure and relative masses of the constituents, ${ }^{2}$ and on increasing the nuasses at constant state all the quantities $\varepsilon, \eta, v, m_{1}, m_{2}, \ldots m_{v}$ increase in the same ratio. The result is a perfectly general expression

$$
\varepsilon^{\prime}=t \eta^{\prime}-p v^{\prime}+\Sigma \mu m^{\prime}
$$

for the energy of the phase, ${ }^{3}$ whose differential must accordingly be

${ }^{\mathrm{I}}$ Gibbs. l.c. I I 9 and thereafter.

${ }^{2}$ Duhem. Le Potentiel Thermodynamique, 33 (1886).

${ }^{3}$ Gibbs l.c. I43. The form in which this operation is executed by Duhem is very interesting. Setting out from the idea of the thermodynamic potential for constant pressure, $\%$, he says essentially that since an $a$-fold total mass of unvaried temperature, pressure and composition will have $a$-fold energy, e11tropy and volume, the function

$$
\zeta=\varepsilon-t \eta+p v
$$

is a honogeneous function of the first degree of the masses of the constituents [though it may indeed, and probably does, involve the ratios of these masses], and therefore from Euler's theorem of homogeneous functions

$$
\begin{gathered}
\zeta=\sum m \frac{\partial \zeta}{\partial m} \\
s-t r+p v=\Sigma u m,
\end{gathered}
$$

since for any constituent $\mu=\partial \zeta / \partial m$, as may readily be shown. 
a general formulation of the differential of the energy, namely

$$
\begin{gathered}
d \varepsilon^{\prime}=t d \eta^{\prime}-p d v^{\prime}+\Sigma \mu d m^{\prime} \\
+\eta^{\prime} d t-v^{\prime} d p+\Sigma m^{\prime} d \mu .
\end{gathered}
$$

But equation (2) is also general, so there follows from (2) and (4)

$$
o=\eta^{\prime} d t-v^{\prime} d p+\Sigma m^{\prime} d \mu
$$

a general relation for all reversible changes of state of the phase. ${ }^{1}$ From this remarkable equation Gibbs had deduced very important results, - the magnificently simple and comprehensive Phase Rule for one thing, ${ }^{2}$ and a series of theorems relating to the vapor pressure curves of liquid mixtures, ${ }^{3}$ to cite no further examples.

Now this deduction of Gibbs, as it stands, is just in so far unsatisfactory as the terms of equation (5) lack interpretation; its correctness indeed is obvious, but its meaning is not. We may have fairly clear ideas of the energy, entropy, volume, temperature and pressure of a phase, of the masses and potentials of its constituents, of the differential of its energy and of the quantities

$$
t d \eta^{\prime} \quad p d i m_{i} \quad \mu_{i} d m_{i}^{\prime}
$$

of heat, work and chemical energy added reversibly to it, - but what does $\eta^{\prime} d t$ denote, or $-v^{\prime} d p$ or $\Sigma m^{\prime} d \mu$, and what is the signification of their algebraic sum appearing in equation (5)? What also is the signification to be attached to the various terms of the second member of (3) ? To seek the interpretation given by the energy theory to these different quantities is the object of the present paper; the results of the investigation should add meaning to the equation (5) and thus make the deductions from it seem more reasonable and therefore more intelligible and satisfactory.

\section{Individual Phases}

A part of the problem has been worked out by Helmholtz in his development of the theory of free energy, where for any thermodynamic system suffering reversible change the quantity $\eta d t$ is looked upon as " the amount of free energy transformed into bound [energy]

${ }^{\mathrm{I}}$ Gibbs, l.c. I43.

${ }^{2}$ Ibid. l.c. I 52.

3Ibid. l.c. 155 . 
during every rise of temperature in the system $1,{ }^{1}$ but this tells us nothing about $v d p$ so it is evident that the whole question must be examined in a somewhat more general way. Let us attend more closely to equation (4)

$$
\begin{gathered}
d \varepsilon^{\prime}=t d \eta^{\prime}-p d v^{\prime}+\Sigma \mu d m^{\prime} \\
+\eta^{\prime} d t-v^{\prime} d p+\Sigma m^{\prime} d \mu .
\end{gathered}
$$

It represents the complete differential of the energy of the phase, relating both to the accession of energy from without in any change and to all possible concomitant changes of energy within the phase. We know the accessions from without to be represented by the first $v+2$ terms of the second member, namely by the first row in the equation as written, and since the total amount of the energy can change only through (positive or negative) accessions from without it follows that the inner rearrangement of the parts of the energy, as these are consequent upon any reversible disturbance of the state of the phase, are represented by the second row of this member and must exactly compensate one another-we must have the Gibbs equation

$$
o=\eta^{\prime} d t-v^{\prime} d p+\Sigma m^{\prime} d \mu ;
$$

the terms of this equation are all energy terms of course. Inner heatings (positive or negative), inner compressions and inner variations in the chenical energies of constituents may result from changes which the phase is, made to undergo, but when these changes are measured by their work equivalents-in energy terms namely-they must balance one another.

The particular signification of the terms in question is obtained perhaps better if the differentiation examined, that of equation (3), be written out in full. We have for the phase in any fixed state

$$
\varepsilon^{\prime}=t \eta^{\prime}-p v^{\prime}+\Sigma \mu m i^{\prime}
$$

which differentiates to

$$
d \varepsilon^{\prime}=\left\{\begin{aligned}
d\left(t \eta^{\prime}\right) & =t d \eta^{\prime}+\eta^{\prime} d t \\
d\left(-p v^{\prime}\right) & =-p d v^{\prime}-v^{\prime} d p \\
d \Sigma \mu m^{\prime} & =\Sigma \mu d m^{\prime}+\Sigma m^{\prime} d \mu
\end{aligned}\right.
$$

${ }^{1}$ Helmholtz. Sitzungsber. Akad. Wiss. Berlin r882. Also Wiss. Abh. 2, 976. Also the very excellent and convenient translation of five of Helmholtz's electrochemical papers, published by the Physical Society of London, page 60 . 
and here it must be noted that the differential of the energy, the sum of the work equivalents of all outer actions, appears as the first column of the right hand member, while the accompanying inner rearrangements of the energy of the phase-which necessarily sum up to zero-appear in the second column. The signification, now, of the integral expressions

$$
t \eta^{\prime} \quad-p v^{\prime} \quad \Sigma \mu m^{\prime}
$$

appears at once from their differentiation. The first of them, $t \eta^{\prime}$, changes uniformly with incoming or outgoing heat, $t d \eta^{\prime}$, and may therefore be looked upon as that portion of the total energy of the phase which is potential with respect to heat,-it may be termed the heat function. The second, - $p v^{\prime}$, changes uniformly with work, - $p d v^{\prime}$, taken up or developed, it is a function which is potential with respect to work, -it is the work function. The third, $\Sigma \mu m^{\prime}$ or $\varepsilon^{\prime}-t \eta^{\prime}+p v^{\prime}$, the (thermodynamic potential) $\zeta^{\prime}$, of these integral quantities is that portion of the energy which changes uniformly with the production or absorption of chemical work, $\Sigma \mu d m^{\prime}$,- it might be termed, for the simple conditions here considered, the chemical function of the phase. Nothing, now, that

$$
\Sigma m^{\prime} d \mu=-\eta^{\prime} d t+v^{\prime} d p
$$

we read from equation (5) that, in any reversible change of state of a pliase : All heat is developed at the expense of the heat function, and this latter is transformed into the work and chemical functions by the amount $-\eta^{\prime} d t$ if the temperature rise; all work is produced at the expense of the work function, which is transformed into the heat and chemical functions by the amount $v^{\prime} d p$ if the pressure rise ; and all chemical work appears at the expense of the chemical function $\zeta^{\prime}$, which is at the same time transformed into the heat and work functions by the amount

$$
\eta^{\prime} d t-v^{\prime} d p=-\Sigma m^{\prime} d \mu
$$

through any variation in the temperature or the pressure. It should be noticed that variations in the relative masses (concentrations) of constituents do not cause variation in the value of the chemical function $\Sigma \mu m^{\prime}$ although they change the values of certain of the potentials; such variations merely effect redistribution among the terms of the sum 


$$
\mu_{r} m_{1}+\mu_{2} m_{2}+\ldots .+\mu_{\nu} m_{\nu}
$$

without altering its total value. The simplification of statement which results from tile introduction of conditions of constant temperature or pressure during change are too apparent to call for separate comment.

The foregoing considerations suffice to give an obvious energy interpretation to the quantities

and to the terms

$$
t \eta^{\prime} \quad-p v^{\prime} \quad \Sigma \mu m^{\prime}
$$

$$
\eta^{\prime} d t \quad-v^{\prime} d p \quad \Sigma m^{\prime} d \mu,
$$

the former are functions potential with respect to the different kinds of action of which the phase is capable, while the latter are the quantities of energy which are transformed from one of these functions to others through concomitant changes in the temperature or pressure. When the total energy of the phase is resolved into pairs of functions, through successive combination of the three just discussed, and these pairs are represented for brevity by the symbols

$$
\begin{aligned}
& \psi^{\prime}=-p v^{\prime}+\Sigma \mu m^{\prime}=\varepsilon^{\prime}-t \eta^{\prime} \\
& \chi^{\prime}=t \eta^{\prime}+\Sigma \mu m^{\prime}=\varepsilon^{\prime}+p v^{\prime} \\
& \varphi^{\prime}=t \eta^{\prime}-p v^{\prime}=\varepsilon^{\prime}-\Sigma \mu m^{\prime},
\end{aligned}
$$

the equation (5) for the differential of the energy takes the following forms :

$$
\begin{aligned}
& d \varepsilon^{\prime}=\left\{\begin{aligned}
d\left(t \eta^{\prime}\right) & = \\
d \psi^{\prime} \eta^{\prime} & +\eta^{\prime} d t \\
d \psi^{\prime} & =-p d v^{\prime} \\
& +\sum \mu d m^{\prime}-\eta^{\prime} d t
\end{aligned}\right. \\
& d \varepsilon^{\prime}=\left\{\begin{aligned}
d\left(-p v^{\prime}\right)= & -p d v^{\prime}-v^{\prime} d p \\
d \chi^{\prime}= & t d \eta^{\prime} \\
& +\Sigma \mu d m^{\prime}+v^{\prime} d p
\end{aligned}\right. \\
& d \varepsilon^{\prime}=\left\{\begin{aligned}
d \Sigma \mu m^{\prime}= & \Sigma \mu d m^{\prime}+\Sigma m^{\prime} d \mu \\
d \phi^{\prime}= & t d \eta^{\prime} \\
& -p d v^{\prime}-\Sigma m^{\prime} d \mu .
\end{aligned}\right.
\end{aligned}
$$

In thermodynamic changes, as described for example, by ( $7 a$ ) all work and chemical energy furnished by the phase are supplied at the 
expense of the function $\psi^{\prime}$ and all heat at the expense of $t \eta^{\prime}$, while, if the temperature change, ' $\psi^{\prime}$ is transformed into $i \eta^{\prime}$ by the amount $\eta^{\prime} d t^{1}{ }^{1}$ Similar interpretations may be given to the forms $\left(7^{b}\right)$ and (7c).

\section{III. (Open Systems) of Phases}

If we consider as our thermodynamic body a system of coexistent phases, instead of a single phase, we shall form an expression for the total energy of the system by summation, over all the different phases, of the equation (3)

$$
\varepsilon^{\prime}=t \eta \gamma^{\prime}-p v^{\prime}+\Sigma \mu m^{\prime}
$$

for the energy of each one. The masses making up the whole are still supposed to be independently variable, - the body is not a (closed, system, it is an open one. The summation gives

$$
\varepsilon=t \eta-p v+\Sigma \mu m
$$

where the unaccented letters refer to the entire mass; for, the temperature, pressure and potentials have uniform values throughout, and the energy, entropy, volume and masses are additive. The differential of the energy is found by a similar summation of

$$
d \varepsilon^{\prime}=t d \eta^{\prime}-p d v^{\prime}+\Sigma \mu d m^{\prime}
$$

as

$$
d \varepsilon=t d \eta-p d v \dashv-\sum \mu d m,
$$

as is indeed obvions anyway since the energy of a heterogeneous system of varying masses subject only to actions of heating and compression is determined by the values of its entropy, its volume and the masses of its constituents-the constituents being as before so chosen that the values of the different $d m$ s are independent. A general expression for the differential of the energy is likewise to be obtained by differentiation of (8), which gives

or, because of (9),

$$
\begin{gathered}
d \varepsilon=t d \eta-p d v+\Sigma \mu d m \\
+\eta d t-v d p+\Sigma m d \mu
\end{gathered}
$$

$$
o=\eta d t-v d p+\Sigma m d \mu
$$

for the entire system. The interpretation of these equations must remain the same as in the more special case of a single phase,-the

${ }^{x}$ Compare Helmholtz, as previously cited, page 60. 
equation (ro) represents the total differential of the energy in all its changes both within and without the system, equation (9) asserts this differential to be the sum of the work equivalents of all outer actions added, whence follows (II) that the inner redistributions of parts of the energy, as consequent upon any reversible disturbance of the state of the system, must compensate one another to maintain the inner equilibrium.

The entire matter may, accordingly, as before be represented in a single expression by writing

$$
d \varepsilon=\left\{\begin{aligned}
d(t \eta) & =t d \eta+\eta d t \\
d(-p v) & =-p d v-v d p \\
d \Sigma \mu m & =\Sigma \mu d m+\Sigma m d \mu
\end{aligned}\right.
$$

where the two vertical columns of the right hand member represent respectively the accessions of energy and the innner rearrangements consequent upon change-the total value of the second column always disappearing. It is seen from the differentiation (12) that the heat function, $t \eta$, of the system is that portion of the total energy which is potential with respect to reversible heat; the work function, $-p v$, is potential with respect to reversible compression-work; while the remainder of the energy, the quantity $\varepsilon-t \eta+p v$ or $\Sigma \mu m$, the chemical function in this particular case, the thermodynamic potential $\zeta=\varepsilon-t \eta+p v$ in general, is transformed into the other two in proportion as the temperature and the pressure suffer change. In any reversible thermodynamic variation of state there occurs a redistribution among these potential functions, such that $\eta d t$ is transferred to the heat function if the temperature change and - vdp to the work function if the pressure change, the algebraic sum of both, $-\Sigma m d \mu$, being contributed by the chemical function to maintain the inner equilibrium.

It may be noted in particular that in a compression the energy - $p d v$ is added to the work function, but the quantity $v d p$ is transferred therefrom to the heat and chemical functions if the pressure rise, - the energy added does not in general increase the work function alone. Similarly, on heating, the energy $t d \eta$ is added to the heat function, but at the same time the quantity ndt is contributed thereto by the other functions to restore the inner equilibrium if the 
temperature rise, but is gained by them if it fall, -the added energy again not coming solely to the good of the function through which the supply of energy to the system is introduced. This is somewhat as though work were added to a strained spring fastened to the piston confining an air cushion in a cylinder; the energy added is not stored wholly in the increased potential energy of the spring, it is transferred in part, through compression, to that of the air cushionto restore the inner equilibrium of the system.

It may be said in general that all addition of heat increases the heat function and all addition of work the work function, while through change of temperature the energy $\eta d t$ is transferred from the chemical function to the heat function and through change of pressure the energy $v d p$ passes from the work function to the chemical one-to the thermodynamic potential $\xi$.

These general relations assume what may possibly be considered a somewhat simpler form when the above potential functions are combined into pairs, as was done in studying the behavior of single phases. We will put

$$
\begin{aligned}
& \psi=-p v+\Sigma \mu m=\varepsilon-t \eta \\
& \chi=t \eta+\Sigma \mu m=\varepsilon+p v \\
& \varphi=t \eta-p v=\varepsilon-\Sigma \mu m
\end{aligned}
$$

and consequently

$$
\begin{array}{r}
d \varepsilon=\left\{\begin{aligned}
d(t \eta)= & t d \eta+\eta d t \\
d \psi= & -p d v \\
& +\Sigma \mu d m-\eta d t
\end{aligned}\right. \\
d \varepsilon=\left\{\begin{aligned}
d(-p v)= & -p d v-v d p \\
d \chi & =\begin{array}{c}
t d \eta \\
+\Sigma \mu d m+v d p
\end{array}
\end{aligned}\right.
\end{array}
$$

while the form with $\varphi$ is best left in the shape (I2) where $d(t \eta)$ and $d(-p v)$ can readily be considered together.

Helmholtz has termed the work and heat functions of (I $4 a$ ) the "free" and "bound" energies of the system for isothermal changes, the former being free for conversion into work while the latter is bound in this respect; the former, namely, is the potential energy with respect to work, the latter is potential with respect to heat only. 
This terminology can be applied to both $\left(\mathrm{I}_{4} a\right)$ and ( $\left.\mathrm{I}^{b} b\right)$. The former asserts that in isothermal changes all work is produced at the expense of the free energy and all heat at the expense of the bound, while free energy is transformed into bound by the amount $\eta d t$ if the temperature rise. This was clearly recognized by Helmholtz ${ }^{1}$ and it supplies in so far the interpretation to be given to the quantities $\eta d t$ and $t \eta$, as was stated by him in his famous first paper on the free energy theory. Equation ( $14 b$ ) asserts on the other hand that in isobaric changes work is produced at the expense of the free energy, $-p v$, and heat at the expense of the bound, ${ }^{2} \chi$, while free energy is transformed into bound by the amount vodp if the pressure rise. This latter conception is, to be sure, in so far more artificial than the former as neither - pv nor $\varepsilon+p v=\chi$ can be considered as positive parts of the total energy of the system, and, too, the (bound, energy is not bound in this case as regards the production of chemical work ; its usefulness nevertheless is no wise impaired by these considerations. The third form of statement, that with $\varphi$, reads that all external actions are produced at the expense of the action function $\varphi$, while the latter is transformed into the chemical function $\Sigma \mu m$ (the energy of the ("air cushion ") by the amount

$$
\Sigma m d \mu=-\eta d t+v d p
$$

through such conconitant variations of temperature or of pressure as may arise.

\section{Closed Systems of Phases}

For closed systems of phases, $i . c$. homogeneous or heterogeneous systems of fixed quantities of substances, the whole matter is simplified by the disappearance of all quantities of chemical work done upon the system or by it. The energy of the entire mass becomes a function of its total entropy and volume simply and, we have, as at first,

$$
d \varepsilon=t d \eta-p d v
$$

The total differential (IO) of the energy may then be written

$$
\begin{aligned}
d \varepsilon & =t d \eta-p d v \\
& +\Sigma \mu d m+\eta d t-v d p+\Sigma m d \mu
\end{aligned}
$$

${ }^{1}$ Helmholtz. 1. c. page 60 of the Physical Society's translation.

"Compare Gibbs, 1. c. I48. 
whence

so that

$$
\begin{gathered}
o=\eta d t-v d p+\Sigma \mu d m+\Sigma m d \mu \\
d \Sigma \mu m=-\eta d t+v d p
\end{gathered}
$$

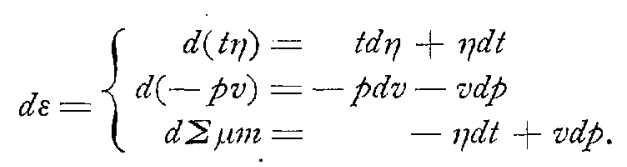

The state of affairs is here simply that in any reversible change all heat is produced from the heat function and all work from the work function, while the chemical function gains -- $\eta d t$ and $v d p$ from the former and the latter through any changes of temperature or pressure which occur; the simile of the air cushion applies with peculiar force to the behavior of the chemical function $\zeta$ in this case. In the more special modes of description employing the functions $\psi$ or $\chi$ we write

$$
\begin{array}{r}
d \varepsilon=\left\{\begin{aligned}
d(t \eta) & =t d \eta+\eta d t \\
d \psi & =-p d v-\eta d t
\end{aligned}\right. \\
d \varepsilon=\left\{\begin{aligned}
& d(-p v)=-p d v-v d p \\
& d \chi= t d \eta+v d p
\end{aligned}\right.
\end{array}
$$

which assert simply that, whether the free and bound energies for isothermal or for isobaric changes be considered, all work done, $-p d v$, is produced from the free energy and all heat, $t d \eta$, fron the bound, while in the former case $\eta d t$ is the energy transferred from the free energy to the bound if the temperature change, and in the latter $v d p$ is that passing from the free to the bound through changes of pressure.

\section{The General Case}

There remains now merely to extend these considerations to the generalized case in which the working system can produce reversible actions other than effects of heating, compression and the transfer of masses, as for example in the production of an electric current. In such a case the state of the system requires for its determination a number of parameters $c_{i}, c_{2}, \ldots$ in addition to the entropy, volume and masses previously considered, and we must write, 


$$
d \varepsilon=t d \eta-p d v+\Sigma \mu d m+\Sigma C d c
$$

where $C_{j}$ is set for the derivative $\partial \varepsilon / \partial r_{j}$ and the second summation extends over all the new parameters. If we differentiate the quantity

$$
\zeta=\varepsilon-t \eta+p v
$$

termed $\zeta$ consistently by Gibbs but written often as $\Sigma \mu m$ in the foregoing special cases, we shall have

$$
d \xi=d \varepsilon-t d \eta+p d v-\eta d t+v d p
$$

and, on comparison with ( 7 ),

$$
d \zeta=\Sigma \mu d m+\Sigma C d c-n d t+v d p .
$$

We can now write

$$
\left\{\begin{aligned}
d(t \eta)= & t d \eta+\eta d t \\
d(-p v)= & -p d v-v d p \\
d \zeta= & \Sigma \mu d m-\eta d t \\
& +\Sigma C d c+v d p
\end{aligned}\right.
$$

which is seen again to be the total differential of the energy of the system. For the first column of the second member is this differential, by (17), and the second is zero. So the interpretation of the quantities

$$
t \eta \quad-p v \quad \zeta \quad-\eta d t \quad v d p
$$

remains in this general case much as in the simpler ones previously studied: All work is supplied by the work function and all heat by the heat function, while through any changes of temperature or pressure which arise there result transfers of $-\eta d t$ and $+v d p$ from the heat and work functions respectively to $\zeta$ - the athermodynamic potential for constant pressure) , of Duhem, the characteristic function $-H^{\prime}$ of Massieu. The thermodynamic potential takes charge of the inner equilibrium of the system.

With use of the potential functions

$$
\begin{aligned}
& \psi=\varepsilon-t \eta \\
& \chi=\varepsilon+p v
\end{aligned}
$$

the equation (Iga) assumes the forms

$$
d \varepsilon=\left\{\begin{aligned}
d(t \eta)= & t d \eta+\eta d t \\
d \psi= & -p d v \\
& +\Sigma \mu d m \\
& +\Sigma C d c-\eta d t
\end{aligned}\right.
$$




$$
d \varepsilon=\left\{\begin{aligned}
d(-p v)= & -p d v-v d p \\
d \chi= & t d \eta \\
& +\Sigma \mu d m \\
& +\Sigma C d c+v d p .
\end{aligned}\right.
$$

From these it appears that, even in this general case, all heat is produced from the bound energy, $t \eta$, and all outer work

$$
\text { outer work }=-p d v+\Sigma \mu d m+\Sigma C d c
$$

from the free energy, $\psi$, while free energy is transformed into bound by the amount $\eta d t$ if the temperature change $;^{1}$ that, further, all heat and non-mechanical forms of work are produced from the bound energy, $\chi$, and all mechanical work from the free, $-p v$, while through changes of pressure the latter is converted into the former by the amount $v d p .^{2}$

So, in every possible case : $\mathrm{I}$. The function

$$
\chi=\varepsilon+p v
$$

is potential with respect to all possible isobaric action save compression work, and it changes by $v d p$ if the pressure change; 2 . The function

$$
\psi=\varepsilon-t \eta
$$

is potential with respect to all possible isothermal work (wholly convertible work equivalents), and it changes by - $\eta d t$ if the temperature change; and 3 . The function

$$
\xi=\varepsilon-t \eta+p v
$$

'Compare Helmholtz, $l$. c.

${ }^{3}$ Helmholtz ( l.c. 59) has termed the entropy "The heat-capacity for heat produced at the expense of the free energy $\zeta^{\prime \prime}$ during adiabatic change $)$. This is indicated directly by ( $(9 b)$, in particular by the formulation

$$
-\frac{\partial \varphi^{\prime}}{\partial t} d t=\eta d t=\frac{\partial(t \eta)}{\partial t} d t .
$$

The analogous remark derived from ( $19 c$ ) and relating to the volume is thatThe volume is the work capacity for compression produced at the expense of the free energy, $-p v$, in adiabatic changes when all work done is compression work. For we note that

$$
-\frac{\partial(-p v)}{\partial p} d p=v d p=\frac{\partial \chi}{\partial p} d p
$$


is potential for all actions save heating and the production of compression work, and it changes with temperature and pressure by the amount

$$
-\eta d t+v d p \text {. }
$$

It may be said, finally, that the energy quantity

$$
\text { ndt }
$$

is in every case transferred through change of temperature from the integral function which is potential with respect to non-mechanical forms of work, to that which is potential for heat; and that the energy

$$
v d p
$$

is always transferred through change of pressure from the function which is potential with respect to mechanical work to that potential with respect to non-mechanical work ${ }^{1}$ (not heat). The former represents the heat produced in the system from its stock of wholly convertible work equivalents and the latter represents compression stored there at the expense of the system's capacity for producing mechanical work. The generality of these relations is perhaps easier seen when the equations (I $9 a$ ) to (Igc) are written out more in full-as follows :

$$
\begin{array}{r}
d \varepsilon=\left\{\begin{aligned}
d(t \eta)= & t d \eta+\eta d t \\
d(-p v)= & -p d v-v d p \\
d \zeta= & \Sigma \mu d m-\eta d t \\
& +\Sigma C d c+v d p
\end{aligned}\right. \\
d \varepsilon=\left\{\begin{aligned}
& d(t \eta)= t d \eta+\eta d t \\
& d \psi=-p d v-v d p \\
&+\Sigma \mu d m-\eta d t \\
&+\Sigma C d c+v^{\prime} d p
\end{aligned}\right.
\end{array}
$$

IMy attention has been caught by a footnote in a paper by L. Natanson, Zcit. phys. Chim. Io, 74r, (1892), where the quantities $y_{d} d t$ and $z^{\prime} d p$ are terned the transformed heat and the iransformed work of the system. The source cited is Natanson's Theoretische Physik, which however has appeared only in Polish and is not accessible to me. It seems probable that this author had in mind some such view as the above. Ifater: See, however, the note at the close of this article. 


$$
d \varepsilon=\left\{\begin{aligned}
d(-p v)= & -p d v-v d p \\
d \chi= & t d \eta+\eta d t \\
& +\Sigma \mu d m-\eta d t \\
& +\Sigma C d c+v d p
\end{aligned}\right.
$$

the statements made are verified in each of the three.

The first (Iga) of these equations has for simplicity of statement and readiness of interpretation much to recommend it, and this may account in some measure for the prominent part taken in recent years by the thermodynanic potential $\zeta$ in the development of the theory of chemical equilibria; the appearance here, too, of the temperature and pressure as independent variables is an advantage not offered by the other forms of statement. The inportant equations of the potential theory, when it is cast into this form, are then (for the case where the differentials $d c$ disappear )

$$
\begin{gathered}
d \xi=\Sigma \mu d m-\eta d t+v d p \\
o=\eta d t-v d p-\Sigma m d \mu,
\end{gathered}
$$

their interpretation is greatly aided by the foregoing considerations.

The view advocated in the present paper is, briefly, that just as

$$
d \varepsilon=t d \eta-p d v+\Sigma \mu d m
$$

represents the change of the energy of a material system, as dependent directly upon actions from without, so is the sum

$$
\eta d t-v d p+\Sigma m d \mu=o
$$

to be regarded as denoting the mutually compensating changes of the energy, which arise within the system itself when actions occur. The individual terms of this sum may then be regarded very suitably as the quantities of energy which are transformed from certain of the potential functions of the system into others during change of temperature or pressure-the potential functions themselves maintaining their general significations thereby. ${ }^{1}$

Cornell University, Deccmber İ'g6.

${ }^{x}$ After this paper was in type the writer discovered very unexpectedly that the general features of the view which it presents are contained in a brief 
article by L. Natanson in Wiedemann's Annalen, 42, 178 (189I), which had been altogether overlooked. It has seemed best all the same, upon due consideration, to allow the paper to appear, and this for the reasons that the more detailed discussion here given may have some value in aiding a comprehension of the matter, that the present statement bears immediately upon certain general formulas of great importance in Gibbs's theory of thermodynamic equilibria-especially upon the interpretation to be given to his very remarkable equation (97), and also because the view in question does not seem to have received the recognition which it deserves-as is evinced by the absence of any employment of it in the text books of van Laar (r893) and of Helm (I894) on the thermodynamics of chemical processes. 